\title{
A New Energy Efficient Data Gathering Approach in Wireless Sensor Networks
}

\author{
Jafar Amiri $^{1}$, Masoud Sabaei ${ }^{2}$, Bahman Soltaninasab ${ }^{1}$ \\ ${ }^{1}$ Department of Computer and IT Engineering, Professional and Technical University of Tabriz, Tabriz, Iran \\ ${ }^{2}$ Department of Computer and IT Engineering, Amirkabir University of Technology, Tehran, Iran \\ Email: \{Jafar_amiri85, Bahman_soltaninasab\}@yahoo.com, sabaei@aut.ac.ir
}

Received November 16, 2010; revised October 30, 2011; accepted November 15, 2011

\begin{abstract}
Data gathering in wireless sensor networks is one of the important operations in these networks. These operations require energy consumption. Due to the limited energy of nodes, the energy productivity should be considered as a key objective in design of sensor networks. Therefore the clustering is a suitable method that used in energy consumption management. For this purpose many methods have been proposed. Between these methods the LEACH algorithm has been attend as a basic method. This algorithm uses distributed clustering method for data gathering and aggregation. The LEACH-C method that is the improvement of LEACH, which performs the clustering in centralized mode. In this method, collecting the energy level of information of every node directly in each period increases the energy cost. Also the phenomenon that is seen by sensor nodes continually change over time. Thereby the information received by nodes is correlated. Sending time correlated data in the network cause to energy dissipation. TINA method and its improvement have been proposed in order to not sending correlated data. These approaches have reported errors. In this paper, the idea of not sending time correlated data of nodes has been considered by using the time series function. Also, a model to estimate the remaining energy of nodes by the base station has been presented. Finally, a method has been proposed to aware the base station from the number of correlated data in each node as the estimation of energy will be more precise. The proposed ideas have been implemented over the LEACH-C protocol. Evaluation results showed that the proposed methods had a better performance in energy consumption and the lifetime of the network in comparison with similar methods.
\end{abstract}

Keywords: Clustering; Sensor Network; Data Correlation; Time Series; Energy Prediction

\section{Introduction}

Wireless sensor networks are a class of wireless ad-hoc networks. In these networks, sensor nodes collect data from physical environment and after processing sent to the base station $\left(\mathrm{BS}^{1}\right)$. Thus allow monitoring and control many types of physical parameters. Each sensor node has limited energy and in most applications, replacing energy sources are not possible. So lifetime of sensor nodes is highly dependent on energy stored in their battery. Clustering is a designing method that used for management of wireless sensor networks. In this method, the network is divided into several independent collections that these collections called cluster. So each cluster contains a number of sensor nodes and a cluster head node. Member nodes in a cluster send their data to relative cluster head node. Cluster head node aggregates these data and send to the base station. Therefore, clustering in sensor networks has advantages such as data aggregation support [1], data gathering facilitation [2], organizing a suitable ${ }^{1}$ Base Station. structure for scalable routing [3], and efficient propagation of data in the network [4].

Data gathering in wireless sensor networks is an important operation in these networks and for this purpose many methods have been proposed. The $\mathrm{LEACH}^{2}$ [5] protocol has been considered as a hierarchical basic method. This method is suitable for monitoring applications. Each node periodically senses the information and sends them. In this algorithm, the clustering method has used for data gathering and aggregation. The cluster and cluster head selected randomly, therefore there is no assurance to select the exact improved number and uniform distribution of cluster head throughout the network. Many improvements in LEACH protocol have been presented. LEACH- $\mathrm{C}^{3}$ method [6] is an example of these improvements. In LEACH-C, the forming of clusters is done using a centralized algorithm by the base station in the starting of each period. Base Station uses the received

\footnotetext{
${ }^{2}$ Low-energy Adaptive Clustering Hierarchy.

${ }^{3}$ LEACH-Centralized.
} 
information from nodes for finding the predetermined number of cluster heads and network configuration within the clusters. This information contains position and energy of nodes. Another improvement to this algorithm is the use of estimation. One of these algorithms is LEACH$\mathrm{CE}^{4}$ [7]. In the proposed technique energy level collected from all nodes in two primary periods but not collected in the other periods. Instead, the average energy of initial periods is used. Considering that the energy estimation in this method is not precise, this clustering scheme is not efficient and suitable. There is some proposed clustering methods that ABCP [8] and ABEE [9] and HMM [10,11] are samples of them.

Each sensor node is observer of a physical phenomenon. Also physical phenomenon such as temperature and... continuously change in time. So the information provided by sensor nodes is dependent on each other. Some algorithms that based on not sending of correlated data are considered. The TINA $^{5}$ [12] algorithm is one of them. In this algorithm the sensor node compares the value of sampled data with previous data, if that be different send it and otherwise goes to sleep mode. The proposed improvement to this algorithm is that sensor node decides to send data with comparing the value of new sample with last reported data [13]. These mentioned algorithms due to error in report, is not suitable. Therefore, a method proposed to increase the accuracy of data reporting. For precise estimation of nodes energy, the base station must be aware of data time correlation. So with existence of data time correlation and using energy estimation of nodes, a method suggested so that the base station can estimate nodes energy precisely. These methods avoid the overhead excess and increase the network lifetime.

The remaining of this article is organized as follows: In Section 2 related works are reviewed. In Section 3 we introduce correlated data algorithm with the energy predicting technique and the hybrid method. Analysis of experiments with existing nodes offered in Section 4, and we finally in Section 5 summarize and discuss the scheme.

\section{Related Work}

\subsection{LEACH}

One of the most famous hierarchical routing protocols based on clustering, is the LEACH protocol. In this method, each cluster members send their data to cluster head. The cluster head aggregate this data and send to the BS. So the communication cost is reduced. Figure 1 describes this concept:

The operation of cluster forming and data transmission

${ }^{4}$ LEACH-C-Estimate.

${ }^{5}$ Temporal Coherency-Aware in-Network Aggregation. in LEACH is done in two phases that these phases shown in Figures 2 and 3:

Setup phase is the stage of forming cluster and cluster head. At this stage, cluster and cluster head randomly selected. After forming the cluster, cluster head propagate TDMA $^{6}$ scheduler to specify the data transfer time to member nodes. Then the steady-state phase started. In the steady-state phase, each member node in cluster send data to the cluster head only in its time slot and at the rest of time pieces for energy conservation goes to sleep mode.

In this method, the cluster head consumes more energy for receiving, processing and directly sending this data to the BS node. So for increasing the life time of the network it is necessary to replace role of cluster head between network nodes. Many improvements over the LEACH method have been provided that in these improvements firstly, as far as possible the best clustering and cluster head selection is done, secondly possible as possible overhead of the protocol is to be reduced. LEACH$\mathrm{C}$ method is an example of these improvements.

\subsection{LEACH-C}

In LEACH-C, clusters forming in the beginning of every period are done, using the centralized algorithm by the base station. The base station uses received information from nodes that includes energy and node status, uses

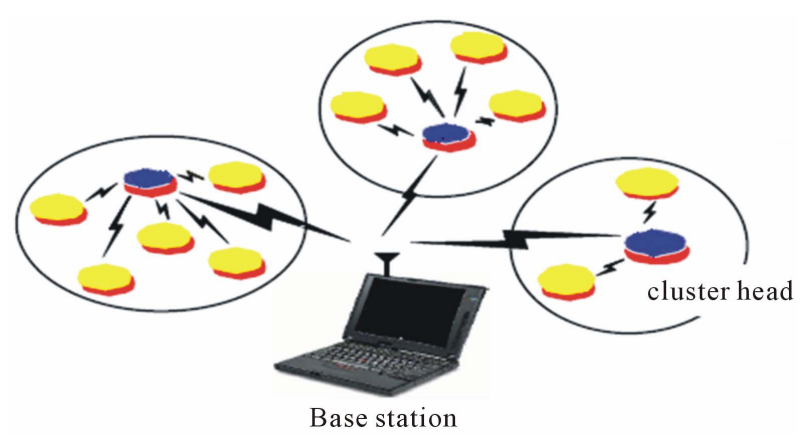

Figure 1. A sensor network with clustering.

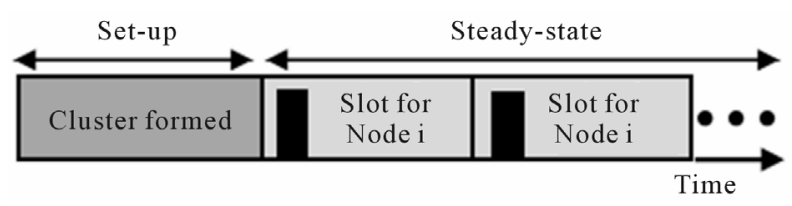

Figure 2. Period of LEACH.

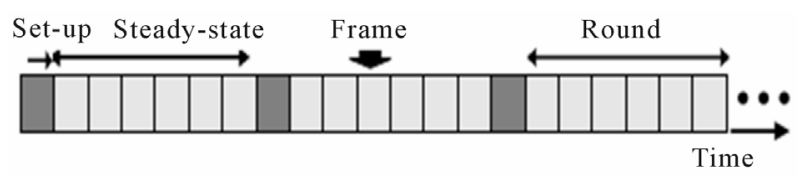

Figure 3. Details of period.

${ }^{6}$ Time Division Multiple Access. 
this information during the setup phase for finding predetermined number of cluster heads and network configuration within the clusters. Next classification of nodes in the clusters is done to minimize energy consumption in order to transfer their data to the related cluster head. Results show that LEACH-C overall performance is better than LEACH because of the optimal forming of clusters by the base station. In addition, the number of cluster heads in each period of LEACH-C is equal to the expected optimal value. While in $\mathrm{LEACH}$ the number of cluster heads varies in different periods because of lack of global coordination.

As in LEACH-C at the beginning of every period energy of nodes must be sent to BS, therefore nodes early discharged and the network lifetime reduces. Another improvement on this algorithm is the use of energy estimation. The LEACH-CE method is an example of these methods.

\subsection{LEACH-CE}

In the LEACH-CE method, the energy level of all nodes collected only in two primary periods and not be collected in other periods. Instead because of knowing information about energy level of nodes, we can calculate energy consumption average for each node by using information of two primary periods. This means that reducing calculated energy level from the energy level of node, causes predicting current energy level of node. The problem of this algorithm is that firstly energy estimation is not done precisely and secondly if nodes have correlated data, while not sending correlated data means that previous data is valid, so this plan of clustering is not suitable and efficient.

\subsection{TINA}

Phenomenon that observed by sensor nodes, continu- ally change in time. Therefore information received by the nodes is correlated on each other. These cases for physical phenomena that are continuous or repetitive, or in an application that the accuracy is not too important, or in a network that node density in a region is high, have seen more. There are two types of data correlation: 1) spatial correlation; 2) Time correlation.

In the spatial correlation, aggregation is done within the network by cluster heads. This is one of the proposed methods to reduce energy consumption. So the nodes that have correlated data send them to cluster head and cluster head after aggregating these data send to the base station. This causes to prevent waste of energy. This method has been implemented in LEACH protocol.

But in the time correlation, each node can have correlated data in successive times. Mohamed and Sharaf proposed the TINA algorithm. The main idea of TINA algorithm was that the sensor nodes send their data only when this data differ with previous data otherwise goes to sleep mode. This algorithm has a reporting error. There is an improvement to this algorithm that presented below.

\subsection{Improvement of TINA}

In this method, the sensor node decides to send data by comparing the value of newly sampled data with last reported data. However, sensor nodes maintain last reported data. For better understanding, we describe this section with an example. Suppose that a given sensor node that received data are $1.0,0.95,1.05,0.95,1.05$ respectively. A threshold has been considered that data changing to this threshold is not important. The value of this threshold considered equal to $10 \%$. First given data that is equal to 1.0 successfully sent and in the next period 0.95 , will not be sent when: $\frac{|0.95-1.0|}{1.0}=5 \%<10 \%$ Otherwise that will be sent. In the third stage $\frac{|1.05-1|}{1}=5 \%<10 \%$ that will not sent and in the fourth stage $\frac{|0.95-1|}{1}=5 \%<10 \%$ that will not sent. This method is suitable when phenomenon changes have not a lot of swing or any special event in the network is done. But as mentioned previously most of phenomenon change continuously with time. So most of data are in ascending or descending mode in the time slices. Or in an application such as temperature for example in a certain time slot occurs a specific event. So the proposed methods have errors and are not suitable. We offer a method to improve this algorithm and prevent the waste of energy. In addition, the problem of data time correlation is not considered in proposed protocols. Therefore, we will check the time correlation of data in the proposed algorithms.

\section{The Proposed Method}

\subsection{Presentation of Methods}

Three ideas are proposed here: 1) the data time correlation; 2) Energy estimation model of nodes and 3) the hybrid method.

In the data time correlation algorithm, Time Series Forecasting method ( $\left.\mathrm{TSF}^{7}\right)$ used to decide sending or not sending of data. Then in time $t$ in the beginning of each period, base station send percentage of error $e(t)$ to all nodes. First data sensed by node and sent. Second and third and fourth data sent based on the improved TINA algorithm. Then the node runs time series function to

\footnotetext{
${ }^{7}$ Time Series Forecasting.
} 
determine the value of prediction of trend line model, to create trend model. In the next times the sensed data compared with predicted value of trend model, if the difference between these two values exceeds a threshold value, data sent to the given node and the node recalculate prediction function of trend model to update the trend line. Otherwise, the sensor node does not send the sensed data with this insurance that sensed data placed in accuracy range of data. So only some data have to be sent that are very different from the trend line model. This help to prevent energy loss.

We call the node energy prediction model LEACH$\mathrm{CEC}^{8}$ and describe as follow. For doing the best clustering, that is needed to know energy of the nodes. The estimation method is a method that has low cost and is suitable. We also use the energy estimation method. For this, we divided LEACH-C protocol to three phases. Topology building phase, setup-state and steady-state. In the first phase nodes send their position to the BS. Then BS creates network topology based on these positions. Once the topology was formed in the base station, base station node calculates the distance of nodes to each other. The BS calculates the amount of energy used in each node in the setup-phase, using a simple mathematical model. Then deduct this amount from primary energy and calculates its remaining energy. Finally do the clustering and goes to the steady-state phase. In this phase for each node, the data time correlated algorithm applied according to the following method.

BS node should be informed of data time correlation in nodes to estimate precisely energy of them. Therefore cluster head create a table that containing list of all members of the cluster. Cluster head registers every node in to the table that have correlated data and do not sent in certain times. In the end of each period, cluster head sends this table with collected data to the base station. This table contains nodes ID and number of times that these nodes not sent data. Base station uses this information for clustering decisions in centralized methods. U1timately that cause to energy estimation in centralized methods is more carefully while the best clustering is created and the network lifetime increases. So in total lifetime of the network, first phase has done once but setup and steady-state phases done as in LEACH-C.

\subsection{Process of Proposed Methods}

\subsubsection{Linear Prediction Method Using Time Series}

Linear prediction method is a powerful technique to predict time series in an environment changing with time. Suppose that you want to contact an independent variable $x$ and a dependent variable $y$ to specify. If we assume that the true relationship between these variables in a straight

\footnotetext{
${ }^{8}$ LEACH-CE-Correlation.
}

line and the value observed for each value of $y$ for every given $x$ is a random variable then we can wrote:

$$
E(y \mid x)=a_{0}+a_{1} x
$$

where in this equation $a_{0}$ is the width from the origin and $a_{1}$ is the slope of the line that is unidentified fixed values. Observed value y can be described with the following equation where the error $\varepsilon$ created because of not conforming real value to the amount of predicted value.

$$
y=a_{0}+a_{1} x+\varepsilon
$$

This pattern is usually named a simple linear regression model. Because that has only one independent variable so that the $x$ independent variables called prediction variable and $y$ called the response variable. Prediction and response variables $x$ and $y$ can be time series in which case we have a time series regression pattern.

There are several methods to estimate unknown parameters $a_{0}, a_{1}$ in Equation (2) that can be used. One method that a lot used is the least square error method in which the $a_{0}, a_{1}$ estimates obtained from minimizing sum of squares errors or remaining's. Suppose we have $\mathrm{n}$ observations of $\left(x_{1}, y_{1}\right),\left(x_{2}, y_{2}\right) \cdots\left(x_{n}, y_{n}\right)$. A model that is based on these observations is written as follows:

$$
y_{i}=a_{0}+a_{1} x_{i}+\varepsilon_{i}, i=1,2, \cdots, n
$$

And the total square error is as the follow:

$$
\phi\left(a_{0}, a_{1}\right)=\sum_{i=1}^{n}\left(y_{i}-a_{0}-a_{1} x_{i}\right)^{2}
$$

So the total square error is simply the total squares of deviations observed $y_{i}$ and $a_{0}+a_{1} x_{i}$. The estimated values of $a_{0}$ and $a_{1}$ that we call them $a_{0}$ and $a_{1}$ that achieved using the least squares method by minimizing $\phi\left(a_{0}, a_{1}\right)$ toward $a_{0}$ and $a_{1}$ so we can write:

$$
\begin{gathered}
\frac{\partial \phi}{\partial a_{0}}=-2 \sum_{i=1}^{n}\left(y_{i}-a_{0}-a_{1} x_{i}\right)=0 \\
\frac{\partial \phi}{\partial a_{1}}=-2 \sum_{i=1}^{n} x_{i}\left(y_{i}-a_{0}-a_{1} x_{i}\right)=0
\end{gathered}
$$

This system of equations called least squares line normal equations system that simplified as the following:

$$
\begin{gathered}
n a_{0}+a_{1} \sum_{i=1}^{n} x_{i}=\sum_{i=1}^{n} y_{i} \\
a_{0} \sum_{i=1}^{n} x_{i}+a_{1} \sum_{i=1}^{n} x_{i}^{2}=\sum_{i=1}^{n} x_{i} y_{i}
\end{gathered}
$$

By solving this system $a_{0}$ and $a_{1}$ estimates or on the other hand $a_{0}$ and $a_{1}$ obtained and so: 


$$
\begin{gathered}
\hat{a}_{1}=\frac{\sum_{i=1}^{n} x_{i} y_{i}-\left[\sum_{i=1}^{n} x_{i}\right]\left[\sum_{i=1}^{n} y_{i}\right] / n}{\sum_{i=1}^{n} x_{i}^{2}-\left[\sum_{i=1}^{n} x_{i}\right]^{2} / n} \\
=\frac{\sum_{i=1}^{n}\left(x_{i}-\bar{x}\right)\left(y_{i}-\bar{y}\right)}{\sum_{i=1}^{n}\left(x_{i}-\bar{x}\right)^{2}}=\frac{s_{x y}}{s_{x x}} \\
\hat{a}_{0}=\bar{y}-\hat{a}_{1} \bar{x}
\end{gathered}
$$

where $\bar{x}=\frac{1}{n} \sum_{i=1}^{n} x_{i}$ and $\bar{y}=\frac{1}{n} \sum_{i=1}^{n} y_{i}$. So the fitted simple linear regression model is as follows:

$$
\bar{y}=\hat{a}_{0}+\hat{a}_{1} x
$$

For each value of the predicted variable $x$ we can obtain corresponding value predicted response from this equation. The fitted values of $\hat{y}_{i}$ corresponding to observed values $\hat{x}_{i}$ for every $i=1,2, \cdots, n$ is the following:

$$
\bar{y}_{i}=\hat{a}_{0}+\hat{a}_{1} x_{i}
$$

The difference between $i$ th given fitted value to an observed yi value called a residue so that:

$$
\hat{e}_{i}=y_{i}-\hat{y}_{i}, i=1,2, \cdots, n
$$

If the fitted model regression for data is appropriate, in this case remains do not follow of appropriate form. There is no clear pattern for the remains.

This method stated by Equation (11) that is a recursive method [9]:

Linear prediction method is a powerful technique for predicting time series in a time-varying environment. This method is expressed in Equation (6) and is a recursive method $[9,10]$ :

$$
y(t+T)=a_{1} y(t)+a_{2} y(t-T)+\cdots+a_{m} y(t-(m-1) T)
$$

Estimated value at time $\mathrm{t}$ as a linear function of previous values in the times " $t-T, t-2 T, \cdots, t-m T$ " has been produced is obtained. In Equation (1) $a_{1}, a_{2}, \cdots, a_{m}$ are the linear prediction coefficients, " $m$ " is the model degree, " $T$ " is the sampling time, $y(t+T)$ is the next observation estimation and $y(t), y(t-T), \ldots, y(t-m T)$ are the present and past observations. The prediction error which is the difference between the predicted and the real values (Equation (7)) must be minimized.

$$
\text { Error }(\%)=\left\{\frac{\text { pridicted value }- \text { Real value }}{\text { Real value }}\right\} * 100 \%
$$

In order to estimate the coefficients of linear prediction model we use the least squares error method and rewrite Equation (6) with considering modeling error in Equation (8):

$$
\begin{aligned}
y(t)= & a_{1} y(t-T)+a_{2} y(t-2 T) \\
& +\cdots+a_{m} y(t-m T)+e(t)
\end{aligned}
$$

The error $e(t)$ is generated because of not adopting the linear prediction model to the real value. So to find the coefficients, $a_{1}, a_{2}, \cdots, a_{m}$ in Equation (8) we use the sum least squares error and set of linear functions. Presented in Equation (9).

$$
\begin{aligned}
& {\left[\begin{array}{c}
y(t) \\
y(t-T) \\
\vdots \\
y(t-m T)
\end{array}\right]=} {\left[\begin{array}{ccc}
y(t) y(t-2 T) & \cdots & y(t-m T) \\
y(t-2 T) & \cdots & y(t-(m+1) T) \\
\vdots & \vdots & \\
y(t-(k+1) T) & \cdots & y(t-(m+k) T)
\end{array}\right] } \\
& *\left[\begin{array}{c}
a_{1} \\
a_{2} \\
\vdots \\
a_{m}
\end{array}\right]+\left[\begin{array}{c}
e(t) \\
e(t-T) \\
\vdots \\
e(t-k T)
\end{array}\right] \\
& Y=\varphi \times A+E
\end{aligned}
$$

Elements in the matrix $A$ are the coefficients which can be found by least squares error method (11):

$$
A=\left(\varphi^{T} \varphi\right)^{-1} \varphi^{T} Y
$$

In Equation (11), $\varphi^{T}$, is the transpose of the matrix $\varphi$, and $\left(\varphi^{T} \varphi\right)$ is the inverse of matrix. In practice:

- If $m$ is chosen larger than is required (i.e. over-estimation of the model order), Equation (11), cannot be solved for any unique set of coefficients, because of some columns in the matrix $\varphi$, are not independent of each other. Hence $\varphi^{T} \varphi$ would be unique and will not have inverse. This means that the system of equations in Equation (8) will have an infinite number of answers for the coefficients. Geometrically speaking, it is like fitting an infinite number of lines to a single point which is not the preferred case.

- If $m$ is chosen less than the required value, the number of independent equations would be more than the number of unknown variables $\left(a_{1}-a_{m}\right)$. Such a system of equations has to be solved for the best approximation of coefficients. The best approximation for coefficients $\left(a_{1}-a_{m}\right)$ is the use of the least squares error method.

Obviously, that is how much precision, the number of the data sent will increase and vice versa.

\subsubsection{The Algorithm Used to Estimate the Energy by Transferring Topology to the Base Station} As previously mentioned, LEACH-C protocol divided to 
three phases: topology building phase, setup-state and steady-state and this algorithm is proposed as following. These three phases is called LEACH-CEC.

\section{a) Topology building phase}

1) Start of network.

2) Base station receives position of all nodes that contain $x$ and $y$.

3) Base station calculates distance of all nodes with each other's using the following Formula (12):

$$
d_{a b}=\sqrt{\left(x_{a}-x_{b}\right)^{2}+\left(y_{a}-y_{b}\right)^{2}}
$$

4) End of phase.

b) Setup-state phase

5) If clustering has changed

5.1) BS calculate energy consumption per node using the Formulae (13) and (14):

$$
E_{T x}(l, d)=\left\{\begin{array}{l}
l E_{\text {elec }}+l \varepsilon_{\text {friss-amp }} d^{2}: d<d_{\text {crossover }} \\
l E_{\text {elec }}+l \varepsilon_{\text {two-ray-amp }} d^{4}: d \geq d_{\text {crossover }}
\end{array}\right.
$$

and

$$
E_{R x}(l)=l E_{\text {elec }}
$$

And after computing, place the sum of two variables in to the $E_{n i}^{t}$.

5.2) Base station calculates remaining energy $\left(E_{R}\right)$ of each node using the Formula (15).

$$
E_{R}=E_{R}-E_{n i}^{t}
$$

5.3) BS audit the information received from $\mathrm{CHs}$, if the nodes name with the number of duplicate data is there, considered in terms of computing nodes remaining energy.

5.4) If $E_{R}<=0$ the node is dead.

5.5) Otherwise the node is alive and participates in Clustering.

6) Clustering is formed.

$E_{n i}^{t}$ : The amount of consumed energy by node $i$ in time $t$.

\section{c) Steady-state phase(Hybrid method)}

7) If node $n$ is a cluster head.

7.1) Cluster head creates a table containing node name and the number of correlated data.

7.2) Cluster head collects data from the members of the cluster.

7.3) If the cluster head has not received data from its member node, registers name of that node in the table and a unit to be added to the number of correlated data. Cluster head sends the table with correlated data to the base station.

8) Otherwise, that is a member node.

8.1) If the node is in turn on then the node runs correlated data algorithm in Figure 4.

8.2) Otherwise goes to sleep mode.

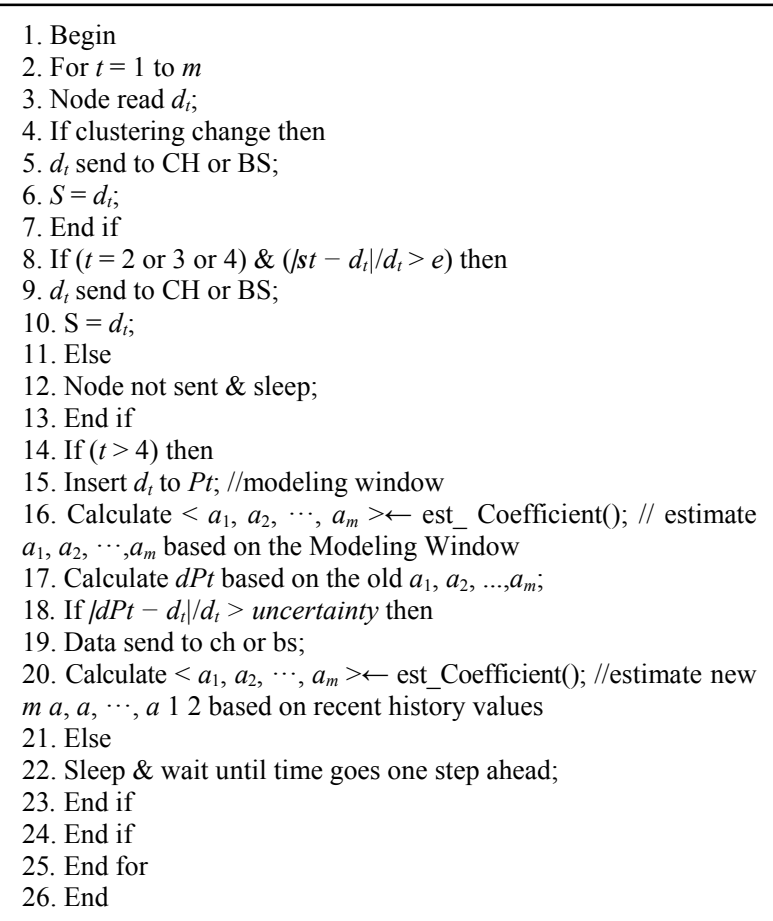

Figure 4. Algorithm of not sending correlated data using TSF.

$E_{T X-a m p}(l, d)$ : Strengthening the energy to transferring 1 bit data in distance $d$.

$\varepsilon_{\text {friss-amp }}$ : Radio energy of amplifier.

$\varepsilon_{\text {two-rav-amp }}$ : Radio energy of amplifier.

$d$ : distance between receiver and sender

$l$ : length of data package.

Table 1 should be created by the cluster head in the steady-state phase. To explain this part, consider a cluster with node numbers 2, 3, 5, 6, 7, 14, and 15 are chosen. Assume that the node 7 is cluster head and the rest is member nodes. If nodes 2 and 5 respectively, each have 2 and 1 correlated data, node 7 as cluster head must create Table 1. This table must be dynamic and at the end of each period must be sent with correlated data.

\section{Simulation and Evaluation of Methods}

\subsection{Simulation Environment}

Simulations have been done on the Redhat9.0 Linux operating system by using NS2 network simulator. LEACH and $\mathrm{LEACH}-\mathrm{C}$ protocols Implementation are from the Uamp project at MIT University on NS2.

Simulations have been done on the dimensions of 100 $\times 100 \mathrm{~m}$ area with 100 sensor nodes. The primary energy of every Node is 2 joule. During our simulations we have chosen some random network topologies to obtain the mean results. Two modes for base station location have been considered. One position in (50 and 50) the exact 
Table 1. Information of node's correlated data in BS.

\begin{tabular}{cc}
\hline Node name & Number of data correlated \\
\hline Node2 & 2 \\
Node5 & 1 \\
\hline
\end{tabular}

center of the network and the latter is in position (50 and 100 ), which is near the area under monitoring. Each period of simulation takes 20 seconds long. Receivers and transmitters follow the model that their parameters are:

$$
\begin{aligned}
E_{\text {elec }}= & 5.0 \times 10^{-8} \mathrm{~J} / \mathrm{bit}, E_{t x}=E_{r x}=5.0 \times 10^{-8} \mathrm{~J} / \mathrm{bit} \\
& \varepsilon_{\text {free-space-amp }}=1.0 \times 10^{-11} \mathrm{~J} / \mathrm{bit} / \mathrm{m}^{2}, \\
& \varepsilon_{\text {two-ray-amp }}=1.3 \times 10^{-15} \mathrm{~J} / \mathrm{bit} / \mathrm{m}^{4}
\end{aligned}
$$

$E_{t x}, E_{r x}$ are Send and receive power needed for each bit. Simulations have done using LEACH, LEACH-C, LEACH-CE and LEACH-CEC protocols.

Simulation assumptions:

1) All nodes are static and have limited resources.

2) Base station has not limited resources.

3) All nodes at any moment have data to send.

4) All Nodes equipped with the location determination

\subsection{The Result of Simulation}

In the NS2 simulator and also LEACH and LEACH-C protocols, data produced with the Uniform distribution. But in fact phenomenon that seen by sensor nodes are continuously changing with time. Therefore, the information received by the sensor nodes is correlated. Therefore generated data by the simulator must have a normal distribution.

Normal distribution Definition: we say a random variable $x$ is normally distributed if its density is as follows:

$$
f_{x}(x)=f_{x}(x ; \mu, \sigma)=\frac{1}{\sqrt{2 \pi \sigma}} \mathrm{e}^{-(x-\mu)^{2}} / 2 \sigma^{2}
$$

where the parameters $\mu$ and $\sigma$ are $-\infty<\mu<+\infty$ and $\sigma>$ 0 . Each distribution with the given density functions as defined in relation (16), called a normal distribution. To show the parameters we have used $\mu$ and $\sigma^{2}$ symbols because we have known that these are the mean and variance parameters of distribution respectively. Figure 5 shows this concept.

For producing data by normal distribution we assume $\mu=0.8$ and $\sigma=1$. In the mentioned protocols data generated in uniform distribution. So we have changed the code of these protocols to normal distribution. In addition, the actual amounts of energy in each node in all protocols of LEACH, LEACH-C, LEACH-CE and
LEACH-CEC in each period was calculated. In our first scenario where the base station along the network are located at the point (50 and 100).

Before reviewing mentioned protocols we first survey the data correlation protocols. We will examine TINA, improvement of TINA Protocols and the idea proposed in conjunction with data correlation (TSF). The produced Data by a node in the algorithms TINA, improvement of TINA and the proposed method (TSF) is reviewed. We took this result that the number of data submissions in TSF method is less than previous methods and the accuracy is high. Figure 6 shows this concept.

We have run each of desired protocols 20 times, so that resulting graphs are the average of results of the runs.

Then we calculated the mean of results and by running data correlation algorithms on them we extracted the following results. We concluded that the number of sent data in TSF method is less than two other methods and have a high accuracy. Error Percentage for correlated data in TSF method is equal to $1 \%$.

Diagram of Figure 6 describes this concept. In a period in specified times we have generated 20 times random data and we repeat it again 20 times. Then we calculated the average of them and have run the data correlation algorithms over them and extracted the following

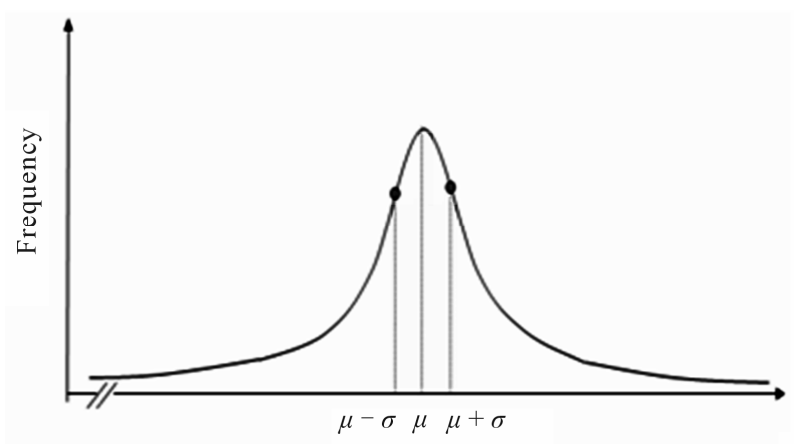

Figure 5. Data generation model in a node.

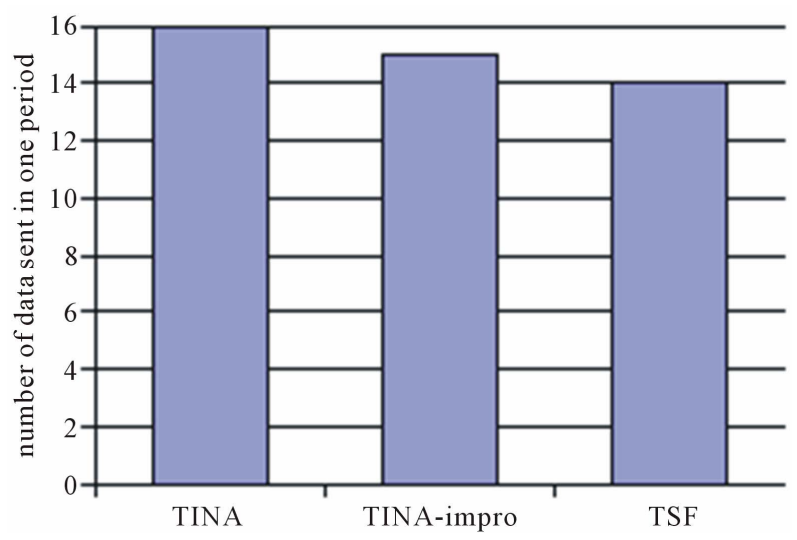

Figure 6. Number of sent data with correlated data. 
results.

In Figure 6 the average of correlated data number in each period with 100 nodes has shown. Until the 300th time in each moment of sending periods at least 6 data are correlated. From 300th to 400th time there is at least 5 correlated data.

As is clear in the Figure 7 the number of correlated data throws down over time. Because of nodes energy level decrease over time and then die. So the probability of existing correlated data will decrease.

\subsection{The First Scenario}

In our first scenario where the base station in points $(50$ and 100) is the network decided Figure 8 shows the amount of energy consumption in each period. In Figure 8, we compared LEACH, LEACH-C, LEACH-CE and the proposed LEACH-CEC protocols and then we have considered the value of correlated data in discussed algo-

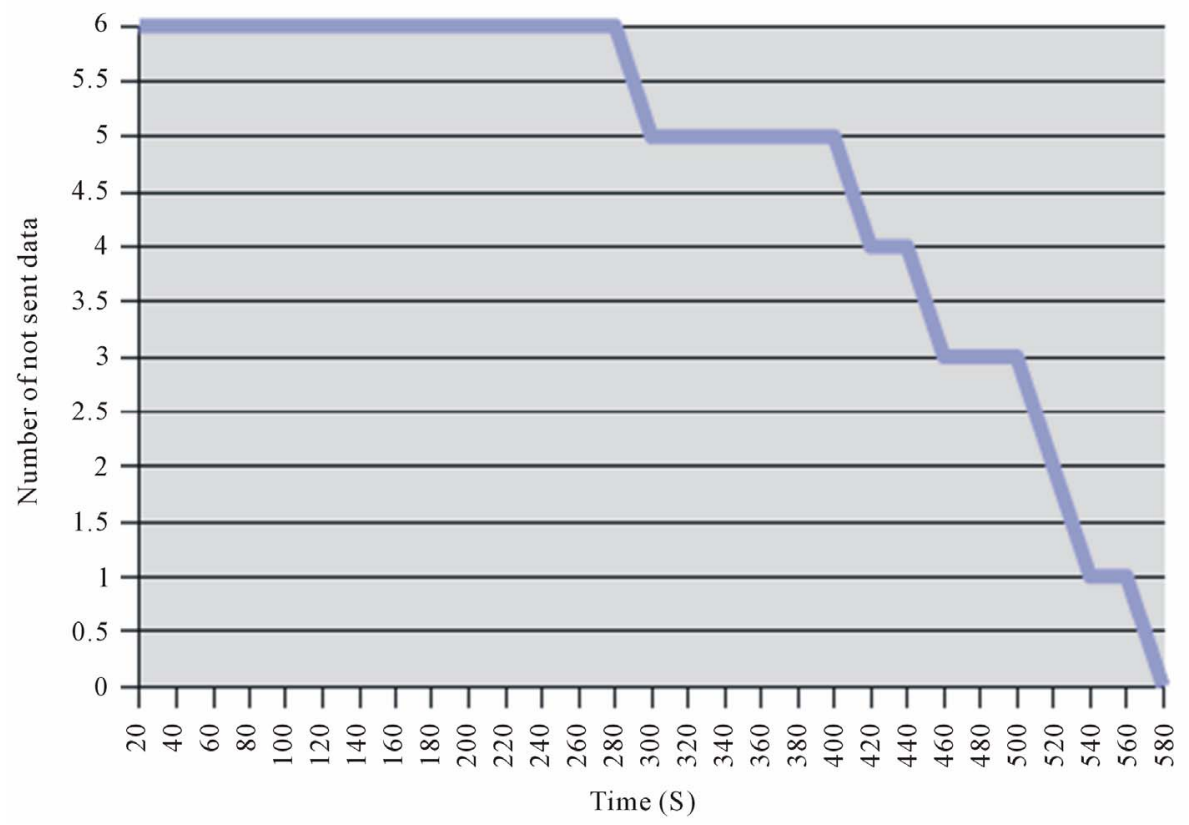

Figure 7. Average number of correlated data in each period.

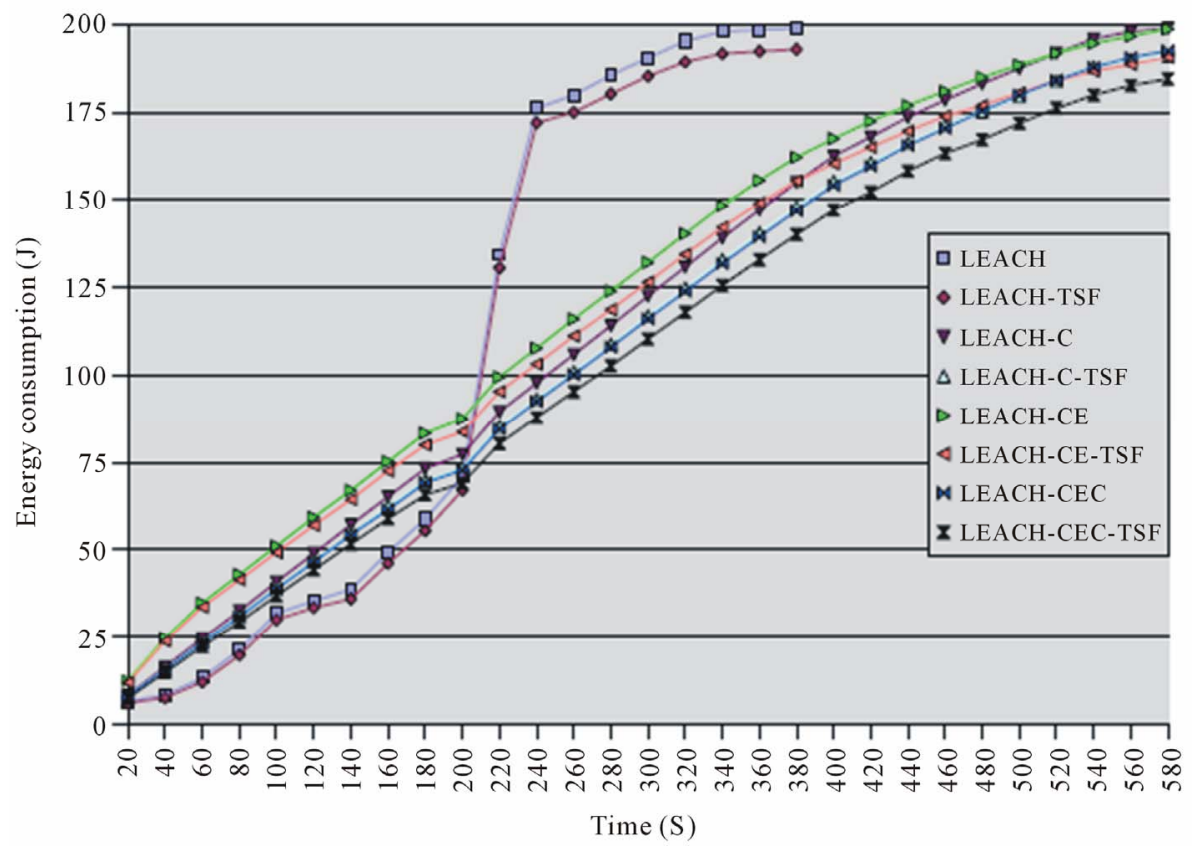

Figure 8. Total energy consumption in the network topology. 
rithms. Then we have obtained the energy consumptions Value for each of protocols. We called them regularly LEACH-TSF, LEACH-C-TSF, LEACH-CE-TSF and LEACH-CEC-TSF. Finally we compared them with each other. We can see the LEACH-CEC-TSF method has better performance than all of mentioned methods.

Figure 9 shows the number of alive nodes at different times. In this Figure 9, 8 methods that mentioned above are assessed by the number of alive nodes in each period. As in Figure 9, in the LEACH-CEC-TSF method the number of alive nodes is more than all other methods. In Figure 9, in the centralized protocols nodes death time has been started from 400 , but in the distributed proto- cols death of nodes has started from 220.

\subsection{The Second Scenario}

In the second scenario the base station's location changed to the point $(50,50)$. Figure 10 shows energy consumption in each period. In this scenario first energy estimation of LEACH-CEC protocol has been compared with the LEACH and LEACH-C and LEACH-CE protocols.

Simulations show that since the base station is located in the center of the network, so the energy consumption is lower in comparison with the first scenario.

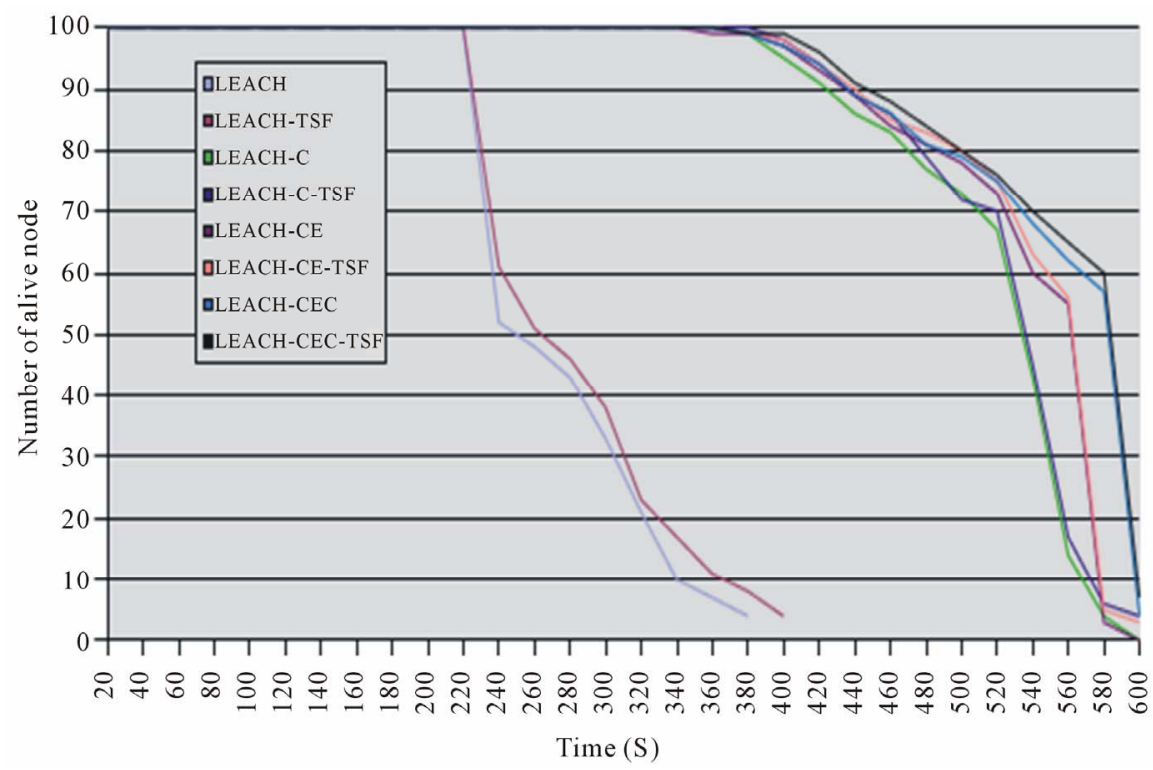

Figure 9. Number of alive nodes in each period.

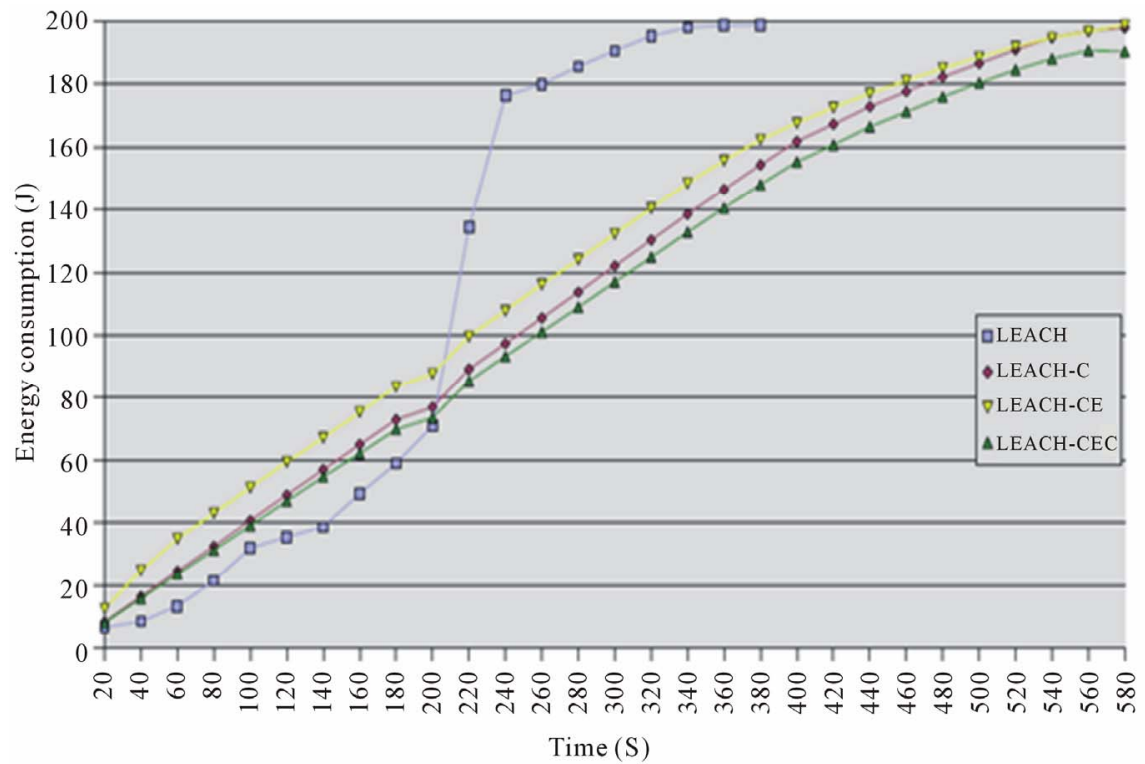

Figure 10. Total energy consumption in the network topology. 
Figure 11 shows data correlation in each of the $\mathrm{LEACH}$ and LEACH-C and LEACH-CE and LEACHCEC protocols. Simulations show that data correlation is not dependent on the particular scenario.

Number of live nodes in each of the LEACH, LEACH-C, LEACH-CE and LEACH-CEC protocols without data correlation is compared in Figure 12.

Applying Data correlation to the each of LEACH, LEACH-C, LEACH-CE and LEACH-CEC protocols shows that live nodes number is further when the base station is located in the center of the network. This operation has shown in Figure 13.

As seen in the distributed protocol, death of nodes started in time 220. But in LEACH, LEACH-C, LEACH$\mathrm{CE}$ and LEACH-CEC centralized protocols death of nodes started in time 360 . As seen the proposed methods have better performance than the previously proposed technique presented. Table 2 shows the percentage of

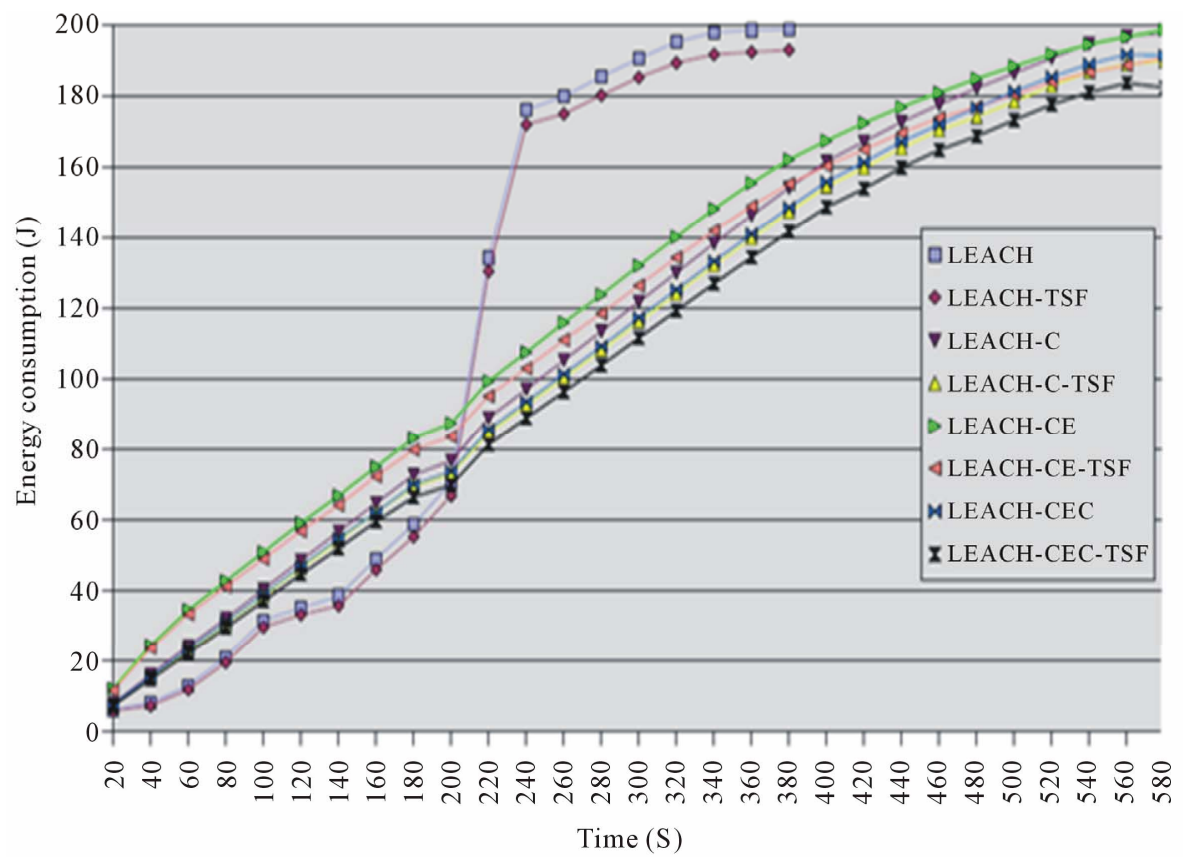

Figure 11. Comparison of energy consumption values by applying data correlation.

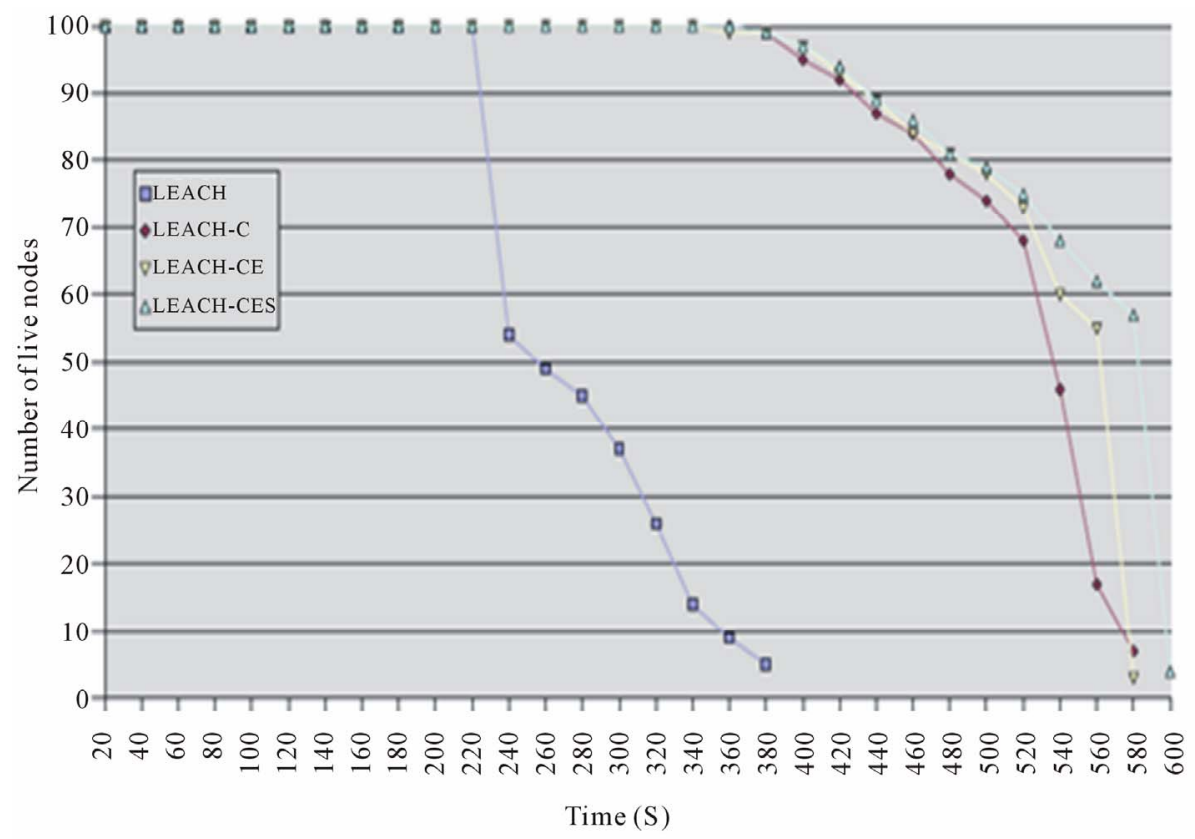

Figure 12. Comparing the number of live nodes. 


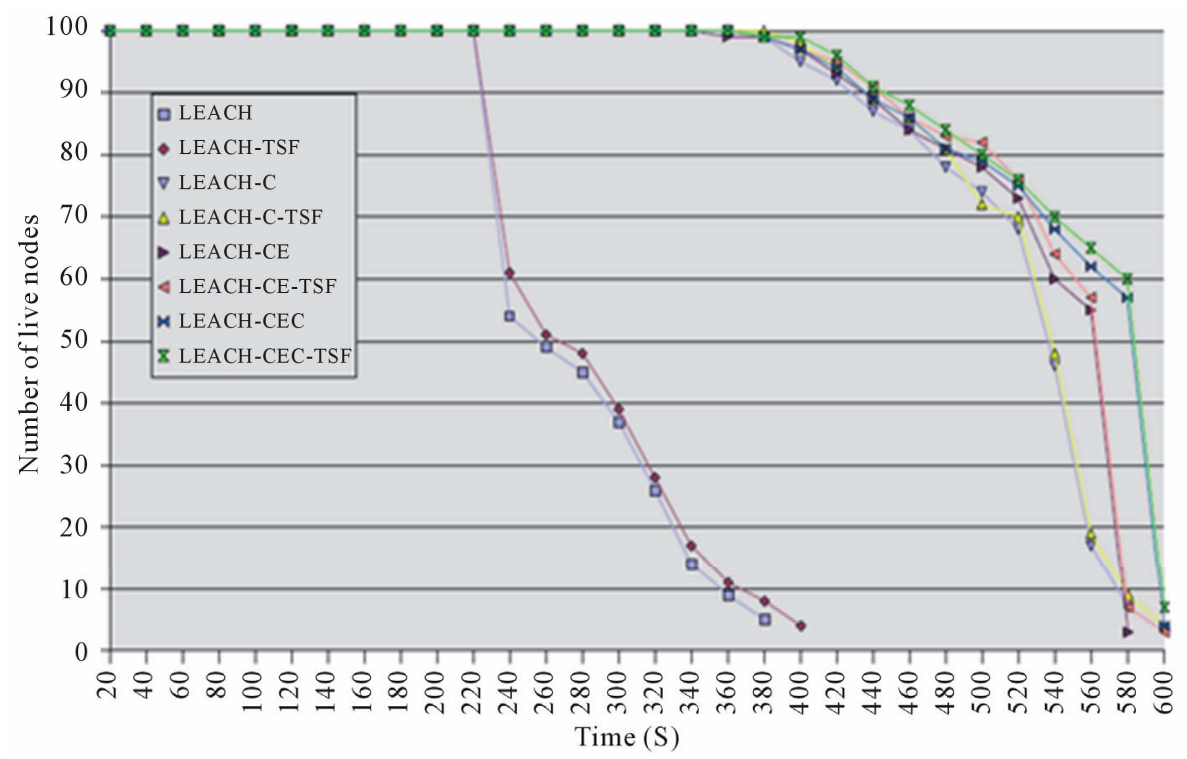

Figure 13. Number of live nodes by applying data correlation.

Table 2. Percentage of energy improvement protocols.

\begin{tabular}{ccccc}
\hline LEACH-CEC-TSF & LEACH-CE-TSF & LEACH-C-TSF & LEACH-TSF & \\
\hline $8 \%$ & $4.5 \%$ & $4 \%$ & $3.1 \%$ & Percentage of improvement in first scenario \\
$8.52 \%$ & $4.82 \%$ & $4.33 \%$ & $3.31 \%$ & Percentage of improvement in second scenario \\
\hline
\end{tabular}

energy improvement by applying TSF to each protocol.

\section{Conclusions}

This article solves the problem of correlated data in all of discussed protocols in this paper. So the nodes that have time correlated data and sending this data wastes their energy and thus network lifetime will decrease. By using the algorithm of data time correlation, the problem will be raised. Also we have eliminated periodic sending of nodes data in LEACH-C protocol. By using energy estimation in LEACH-CEC method there is no need for nodes to send their energy level and position to the base station. They only have to send their position at the beginning of network to the base station and the base station creates network topology and using a simple mathematical calculation will calculate the energy of nodes.

Totally we improved the lifetime of network by using simulation in LEACH, LEACH-C, LEACH-CE and proposed LEACH-CEC protocols. Also we improved energy consumption by using estimation methods. In the future works we will try to use classification of nodes distances scheme to estimate energy of nodes more precisely.

\section{REFERENCES}

[1] S. Bandyopadhyay and E. Coyle, "An Energy Efficient Hierarchical Clustering Algorithm for Wireless Sensor
Networks," Proceedings of IEEE INFOCOM, San Francisco, 30 March 2003.

[2] M. Ali and S. K. Ravula, "Real-Time Support and Energy Efficiency in Wireless Sensor Networks," School of Information Science, Computer and Electrical Engineering Halmstad University, Halmstad, 2008.

[3] A. A. Abbasi and M. Younis, "A Survey on Clustering Algorithms for Wireless Sensor Networks," Elsevier B.V, Amsterdam, 2007.

[4] M. Demirbas and H. Ferhatosmanoglu, "Peer-to-Peer Spatial Queries in Sensor Networks," Proceeding of 3rd IEEE International Conference on Peer-to-Peer Computing ( $p 2 p$ '03), Linköpings, 11 August 2003, pp. 32-39.

[5] W. B. Heinzelman, A. P. Chandrakasan and H. Balakrishnan, "An Application-Specific Protocol Architecture for Wireless Microsensor Networks," IEEE, Vol. 1, No. 4, 2002, pp. 660-670.

[6] S. D. Muruganathan and D. C. F. Ma, "A Centralized Energy-Efficient Routing Protocol for Wireless Sensor Networks," IEEE, Vol. 43, No. 3, 2005, pp. 8-13.

[7] W. B. Heinzelman, A. P. Chandrakasan and H. Balakrishnan, "Application-Speci_c Protocol Architectures for Wireless Networks," IEEE Transactions on Wireless Communications, Vol. 1, No. 4, 2002, pp. 1-154. doi:10.1109/TWC.2002.804190

[8] X. Wang, J.-J. Ma, S. Wang and D.-W. Bi, "Time Series Forecasting for Energy-Efficient Organization of Wireless Sensor Networks," MDPI, 2011. www.mdpi.org/sensors. 
[9] W.-P. Chen, J. C. Hou and L. Sha, "Dynamic Clustering for Acoustic Target Tracking in Wireless Sensor Networks," Proceedings of the 11th IEEE International Conference on Network Protocols, Atlanta, 4-7 November 2003, pp. 284-294. doi:10.1109/ICNP.2003.1249778

[10] C.-K. Liang, Y.-J. Huang and J.-D. Lin, "An Energy Efficient Routing Scheme in Wireless Sensor Networks," 22nd International Conference on Advanced Information Networking and Applications, IEEE, Singapore, 22-25 March 2008.

[11] P. Hu, Z. Zhou, Q. Liu and F. M. Li, "The HMM-Based Modeling for the Energy Level Prediction in Wireless
Sensor Networks," Proceeding of the 2007 2nd IEEE Conference on Industrial Electronics and Applications, Harbin, 23-25 May 2007.

[12] A. S. Mohamed and B. Jonathen, "TINA: A Scheme for Temporal Coherency-Aware In-Network Aggregation," Proceedings of the 3rd ACM International Workshop on Data Engineering for Wireless and Mobile Access, San Diego, 19 September 2003, pp. 69-76.

[13] X. H. Dai, F. Xia and Z. Wang, "An Energy-Efficient In-Network Aggregation Query Algorithm for WSN," IEEE, Beijing, 30 August 2006, pp. 255-258. 